\title{
How to obtain an equitable optimal fair division
}

\author{
Jerzy Legut ${ }^{1}$ \\ Published online: 18 September 2018 \\ (c) The Author(s) 2018
}

\begin{abstract}
A nonlinear programming method is used for finding an equitable optimal fair division of the unit interval $[0,1)$ among $n$ players. Players' preferences are described by nonatomic probability measures $\mu_{1}, \ldots, \mu_{n}$ with density functions having piecewise strict monotone likelihood ratio property. The presented algorithm can be used to obtain also an equitable $\varepsilon$-optimal fair division in case of measures with arbitrary differentiable density functions. An example of an equitable optimal fair division for three players is given.
\end{abstract}

Keywords Fair division - Cake cutting - Optimal partitioning of a measurable space

\section{Introduction}

Suppose we are given a cake to be divided among $n$ players. Let the measurable space $\{[0,1), \mathcal{B}\}$ represent the cake and nonatomic probability measures $\mu_{1}, \ldots, \mu_{n}$ defined on the $\sigma$-algebra of Borel measurable sets describe individual preferences of each player. The measures $\mu_{1}, \ldots, \mu_{n}$ are used by the players to evaluate the size of sets $A \in \mathcal{B}$. Denote by $I=\{1, \ldots, n\}$ the set of numbered players. By an ordered partition $P=\left\{A_{i}\right\}_{i=1}^{n}$ of the cake among the players $i \in I$, is meant a collection of $\mathcal{B}$-measurable disjoint subsets $A_{1}, \ldots, A_{n}$ of $[0,1)$ whose union is $[0,1)$. Let $\mathscr{P}$ stand for the set of all measurable partitions $P=\left\{A_{i}\right\}_{i=1}^{n}$ of $[0,1)$. The problem of fair division of the cake is the task to divide $[0,1)$ among the players $i \in I$, in a way that would be "fair" according to some fairness notions accepted by all players. In classic fair division problem we are interested in giving the $i$-th person a set $A_{i} \in \mathcal{B}$ such that $\mu_{i}\left(A_{i}\right) \geq 1 / n$ for $i \in I$. A simple and well-known method for realizing a fair division (of a cake) for two players is "for one to cut, the other to choose". Steinhaus in 1944 asked whether the fair procedure could be found for dividing a cake among $n$ participants for $n>2$. He found a solution for $n=3$ and Knaster (1946) showed that the solution for $n=2$ could be extended to arbitrary $n$. In the literature several notions of fair divisions $P=\left\{A_{i}\right\}_{i=1}^{n} \in \mathscr{P}$ are discussed

- Proportional division: $\mu_{i}\left(A_{i}\right) \geq 1 / n$ for all $i \in I$.

- Envy-free division: $\mu_{i}\left(A_{i}\right) \geq \mu_{i}\left(A_{j}\right)$ for all $i, j \in I$.

Jerzy Legut

Jerzy.Legut@pwr.wroc.pl

1 Faculty of Pure and Applied Mathematics, Wrocław University of Science and Technology, 50-370 Wrocław, Poland 
- Exact division: $\mu_{i}\left(A_{j}\right)=1 / n$ for all $i, j \in I$.

- Equitable division: $\mu_{i}\left(A_{i}\right)=\mu_{j}\left(A_{j}\right)$ for all $i, j \in I$.

An interesting challenge in fair division theory is to find the best possible equitable partition.

For $X \in \mathcal{B}$ denote by $\mathscr{P}_{e}(X)$ the set all of measurable equitable partitions $P=\left\{A_{i}\right\}_{i=1}^{n}$ of $X$.

Definition 1 The optimal value $\delta(X)$ of the fair division problem of $X \in \mathcal{B}$ is defined by

$$
\delta(X):=\sup \left\{\mu_{k}\left(A_{k}\right): P=\left\{A_{i}\right\}_{i=1}^{n} \in \mathscr{P}_{e}(X)\right\} .
$$

Definition 2 A partition $P^{*}=\left\{A_{i}^{*}\right\}_{i=1}^{n} \in \mathscr{P}$ is said to be an equitable optimal fair division if for all $k \in I$

$$
\delta([0,1))=\mu_{k}\left(A_{k}^{*}\right) .
$$

The equitable optimal fair division will be also called, interchangeably an optimal partition of the measurable space $\{[0,1), \mathcal{B}\}$. For simplicity denote $\delta:=\delta([0,1))$.

Definition 3 A partition $P^{\varepsilon}=\left\{A_{i}^{\varepsilon}\right\}_{i=1}^{n} \in \mathscr{P}$ is said to be an equitable $\varepsilon$-optimal fair division if for all $i \in I$

$$
\mu_{i}\left(A_{i}^{\varepsilon}\right)>\delta-\varepsilon
$$

The existence of optimal partitions follows from a theorem of Dvoretzky et al. (1951):

Theorem 1 If $\mu_{1}, \ldots, \mu_{n}$ are nonatomic countably additive finite measures defined on the measurable space $\{[0,1), \mathcal{B}\}$ then the range $\vec{\mu}(\mathscr{P})$ of the mapping $\vec{\mu}: \mathscr{P} \rightarrow \mathbb{R}^{n}$ defined by

$$
\vec{\mu}(P)=\left(\mu_{1}\left(A_{1}\right), \ldots, \mu_{n}\left(A_{n}\right)\right), P=\left\{A_{i}\right\}_{i=1}^{n} \in \mathscr{P},
$$

is convex and compact in $\mathbb{R}^{n}$.

Finding equitable optimal partitions for arbitrary probability measures is not easy. In the literature of the fair division field there are known some results concerning algorithms of finding such partitions. Legut and Wilczyński (2012) showed how to obtain the optimal partitions for two players. Dall' Aglio and Luca (2014) presented a method for finding maxmin allocations (optimal partitions) for measures satisfying three assumptions:

- complete divisibility of the good (nonatomless),

- mutual absolutely continuity,

- relative disagreement.

Under these assumptions they proposed a subgradient algorithm for obtaining the optimal value of a fair division problem. This algorithm is based on an iterative approximation of the solution up to a given precision and also finds an almost optimal partition. In this paper we require stronger assumptions on the measures. We assume that the density functions of the measures satisfy strictly monotone likelihood ratio property separately on each subintervals of some finite partition of $[0,1)$. In our approach to obtaining equitable optimal division we find accurate equitable optimal partitions using a nonlinear programming method.

Dall'Aglio et al. (2015) presented an algorithm for finding an optimal partition in case of measures defined by densities being simple functions. This result was generalized by Legut (2017) for the case of measures with piecewise linear density functions. The first estimation of the optimal value $\delta$ was given by Elton et al. (1986) and further by Legut (1988). An 
interesting algorithm for finding the bounds for the optimal value was found by Dall'Aglio and Luca (2015).

A general form of the optimal partitions could be helpful in some cases for finding constructive methods of optimal partitioning of a measurable space. Let $S=\left\{\vec{s}=\left(s_{1}, \ldots, s_{n}\right) \in\right.$ $\left.\mathbb{R}^{n}, s_{i} \geq 0, i \in I, \sum_{i=1}^{n} s_{i}=1\right\}$ be an $(n-1)$-dimensional simplex. We can assume that all nonatomic measures $\mu_{1}, \ldots, \mu_{n}$ are absolutely continuous with respect to the same measure $v\left(\right.$ e.g. $v=\sum_{i=1}^{n} \mu_{i}$ ). Denote by $f_{i}=d \mu_{i} / d v$ the Radon-Nikodym derivatives, i.e.

$$
\mu_{i}(A)=\int_{A} f_{i} d \nu, \text { for } A \in \mathcal{B} \text { and } i \in I .
$$

For $\vec{p}=\left(p_{1}, \ldots, p_{n}\right) \in S$ and $i \in I$, define the following measurable sets

$$
\begin{gathered}
B_{i}(\vec{p})=\bigcap_{k=1, k \neq i}^{n}\left\{x \in[0,1): p_{i} f_{i}(x)>p_{k} f_{k}(x)\right\}, \\
C_{i}(\vec{p})=\bigcap_{k=1}^{n}\left\{x \in[0,1): p_{i} f_{i}(x) \geq p_{k} f_{k}(x)\right\} .
\end{gathered}
$$

Legut and Wilczyński (1988) using a minmax theorem of Sion (cf. Aubin 1980) proved the following theorem presented here in less general form

Theorem 2 There exists a point $\overrightarrow{p^{*}} \in S$ and a corresponding equitable optimal partition $P^{*}=\left\{A_{i}^{*}\right\}_{i=1}^{n}$ satisfying

(i) $B_{i}\left(\overrightarrow{p^{*}}\right) \subset A_{i}^{*} \subset C_{i}\left(\overrightarrow{p^{*}}\right)$,

(ii) $\mu_{1}\left(A_{1}^{*}\right)=\mu_{2}\left(A_{2}^{*}\right)=\ldots=\mu_{n}\left(A_{n}^{*}\right)$.

Moreover, any partition $P^{*}=\left\{A_{i}^{*}\right\}_{i=1}^{n}$ which satisfies (i) and (ii) is equitable optimal.

\section{Main result}

In this section we present an algorithm for obtaining an equitable optimal fair division. Suppose we are given $n$ nonatomic probability measures $\mu_{i}, i \in I$, defined on the measurable space $\{[0,1), \mathcal{B}\}$. We need the following

Assumption 1 The measures $\mu_{i}, i \in I$, are absolutely continuous with respect to the Lebesgue measure $\lambda$ defined on $\{[0,1), \mathcal{B}\}$ and additionally

$$
\operatorname{supp}\left(\mu_{i}\right)=[0,1), \quad i \in I .
$$

Let $f_{i}, i \in I$, denote the Radon-Nikodym derivatives of the measures $\mu_{i}$ with respect to the Lebesgue measure $\lambda$. Define absolutely continuous and strictly increasing functions $F_{i}:[0,1] \rightarrow[0,1]$ by

$$
F_{i}(t)=\int_{[0, t)} f_{i} d \lambda, \quad t \in[0,1], \quad i \in I .
$$

For proving our main result we need yet another crucial assumption.

Assumption 2 There exists a partition $\left\{\left[a_{j}, a_{j+1}\right)\right\}_{j=1}^{m}$ of the interval $[0,1)$, where $a_{1}=$ $0, a_{m+1}=1$, such that the densities $f_{i}$ satisfy strictly monotone likelihood ratio (SMLR) property on each interval $\left[a_{j}, a_{j+1}\right)$, 
$j \in J:=\{1, \ldots, m\}$, i.e. for any $i, k \in I, i \neq k$, the ratios $\frac{f_{i}(x)}{f_{k}(x)}$ are strictly monotone on each interval $\left[a_{j}, a_{j+1}\right)$.

Proposition 1 If the density functions $f_{i}, i \in I$, are differentiable and the set

$$
D:=\left\{x \in(0,1): f_{i}^{\prime}(x) f_{k}(x)=f_{i}(x) f_{k}^{\prime}(x), i, k \in I, i \neq k\right\}
$$

is finite then Assumption 2 is satisfied.

Proof Let $D=\left\{a_{2}, a_{3}, \ldots, a_{m}\right\}$ with $a_{1}=0<a_{2}<\cdots<a_{m}<a_{m+1}=1$. It is easy to verify that the set $D$ consists of all the points in which the derivatives

$$
\left(\frac{f_{i}(x)}{f_{k}(x)}\right)^{\prime}, i, k \in I, i \neq k,
$$

change their signs. Then for given $j \in J$ and for all $x \in\left[a_{j}, a_{j+1}\right)$ the derivatives (3) are positive or negative. It means that the ratios $\frac{f_{i}(x)}{f_{k}(x)}$ are strictly monotone on the interval $\left[a_{j}, a_{j+1}\right)$ and the proof is complete.

If the densities $f_{i}, i \in I$, are pairwise different polynomial functions of positive degree, the assumptions of Proposition 1 are obviously satisfied. Consider the problem of the equitable optimal fair division for two players with the following density functions $f_{1}(x)=x \sin \frac{1}{x}+c$, with the constant $c$ satisfying $\int_{0}^{1} f_{1}(x) d x=1$, and $f_{2}(x) \equiv 1$ for $x \in[0,1)$. It is easy to verify, that in this case the set $D$ is infinite.

For proving the main result of this paper we need the following crucial proposition:

Proposition 2 Assumption 2 is satisfied for the densities $f_{i}$ if and only if for any numbers $\theta_{1}, \theta_{2}$ satisfying $a_{j} \leq \theta_{1}<\theta_{2}<a_{j+1}, j \in J$, and any $i, k \in I, i \neq k$ one of the two following inequalities

$$
\begin{aligned}
& \frac{F_{i}(t)-F_{i}\left(\theta_{1}\right)}{F_{i}\left(\theta_{2}\right)-F_{i}\left(\theta_{1}\right)}<\frac{F_{k}(t)-F_{k}\left(\theta_{1}\right)}{F_{k}\left(\theta_{2}\right)-F_{k}\left(\theta_{1}\right)} \\
& \frac{F_{i}(t)-F_{i}\left(\theta_{1}\right)}{F_{i}\left(\theta_{2}\right)-F_{i}\left(\theta_{1}\right)}>\frac{F_{k}(t)-F_{k}\left(\theta_{1}\right)}{F_{k}\left(\theta_{2}\right)-F_{k}\left(\theta_{1}\right)}
\end{aligned}
$$

holds for each $t \in\left(\theta_{1}, \theta_{2}\right)$.

The inequalities (4) and (5) mean that there is a strict relative convexity relationship between the functions $F_{i}$ and $F_{k}, i \neq k$, defined by (1). If the inequality (4) holds, then $F_{i}$ is strictly convex with respect to $F_{k}$. This property is equivalent to the strict convexity of the composite function $F_{i} \circ F_{k}^{-1}$ on the interval $\left(F_{k}\left(a_{j}\right), F_{k}\left(a_{j+1}\right)\right)$ (cf. Palmer 2003). It follows from a result of Shisha and Cargo (1964) (Theorem 1) that $F_{i} \circ F_{k}^{-1}$ is strictly convex on $\left(F_{k}\left(a_{j}\right), F_{k}\left(a_{j+1}\right)\right)$ if and only if the ratio $\frac{f_{i}(x)}{f_{k}(x)}$ is strictly increasing on $\left(a_{j}, a_{j+1}\right)$, which implies Proposition 2.

The relative convexity is one of many various generalizations of convexity started in 1931 by Jessen (1931). They were developed by Popoviciu (1936) and Beckenbach (1937) and continued later by Karlin and Novikoff (1963) especially for applications in approximation theory.

The relation of strict relative convexity induces on each interval $\left(a_{j}, a_{j+1}\right)$ a strict partial ordering of the functions $F_{i}$ (cf. Palmer 2003). Let $F_{i} \prec_{j} F_{k}$ denote that $F_{i}$ is strictly convex with respect to $F_{k}$ on $\left(a_{j}, a_{j+1}\right)$. For each $j \in J$ define permutation $\sigma_{j}: I \longrightarrow I$, such that 


$$
F_{\sigma_{j}(k+1)} \prec_{j} F_{\sigma_{j}(k)},
$$

for $k=1, \ldots, n-1$. Hence for all $t \in\left(a_{j}, a_{j+1}\right)$ we have

$$
\frac{F_{\sigma_{j}(k+1)}(t)-F_{\sigma_{j}(k+1)}\left(a_{j}\right)}{F_{\sigma_{j}(k+1)}\left(a_{j+1}\right)-F_{\sigma_{j}(k+1)}\left(a_{j}\right)}<\frac{F_{\sigma_{j}(k)}(t)-F_{\sigma_{j}(k)}\left(a_{j}\right)}{F_{\sigma_{j}(k)}\left(a_{j+1}\right)-F_{\sigma_{j}(k)}\left(a_{j}\right)}
$$

The following theorem presents an algorithm for obtaining an equitable optimal fair division.

Theorem 3 Let a collection of numbers $z^{*},\left\{x_{k}^{*(j)}\right\}, k=1, \ldots, n-1, j \in J$, be a solution of the following nonlinear programming $(N L P)$ problem

$\max z$

subject to constraints

$$
z=\sum_{j=1}^{m}\left[F_{i}\left(x_{\sigma_{j}(i)}^{(j)}\right)-F_{i}\left(x_{\sigma_{j}(i)-1}^{(j)}\right)\right] \quad i=1, \ldots, n,
$$

with respect to variables $z,\left\{x_{k}^{(j)}\right\}, k=1, \ldots, n-1, j \in J$, satisfying the following inequalities

$$
\begin{gathered}
0=a_{1} \leq x_{1}^{(1)} \leq \cdots \leq x_{n-1}^{(1)} \leq a_{2} \\
a_{2} \leq x_{1}^{(2)} \leq \cdots \leq x_{n-1}^{(2)} \leq a_{3} \\
\quad \cdots \\
a_{m} \leq x_{1}^{(m)} \leq \cdots \leq x_{n-1}^{(m)} \leq a_{m+1}=1
\end{gathered}
$$

Then the partition $\left\{A_{i}^{*}\right\}_{i=1}^{n} \in \mathscr{P}$ of the unit interval $[0,1)$ defined by

$$
A_{i}^{*}=\bigcup_{j=1}^{m}\left[x_{\sigma_{j}(i)-1}^{*(j)}, x_{\sigma_{j}(i)}^{*(j)}\right), i \in I,
$$

where $x_{0}^{*(j)}=a_{j}, x_{n}^{*(j)}=a_{j+1}, j \in J$, is an equitable optimal fair division for the measures $\mu_{i}, i \in I$.

If for some $i \in I$ and $j \in J$, the equality $x_{\sigma_{j}(i)-1}^{*(j)}=x_{\sigma_{j}(i)}^{*(j)}$ holds we set $\left[x_{\sigma_{j}(i)-1}^{*(j)}, x_{\sigma_{j}(i)}^{*(j)}\right)=\emptyset$ in the union of intervals (10). We need first to prove the following

Lemma 1 Let $\left\{A_{i}^{0}\right\}_{i=1}^{n} \in \mathscr{P}$ be an equitable optimal fair division of the unit interval $[0,1)$. Suppose that for two players $i_{1}, i_{2} \in I$, and $j \in J$, there exist numbers $c, d$, e with $a_{j} \leq$ $c<d<e<a_{j+1}$ such that

$$
[c, d) \subset A_{i_{1}}^{0} \text { and }[d, e) \subset A_{i_{2}}^{0},
$$

then

$$
\sigma_{j}^{-1}\left(i_{1}\right)<\sigma_{j}^{-1}\left(i_{2}\right)
$$

Proof For simpler notation we set $i_{1}=1$ and $i_{2}=2$. Suppose that the inequality (11) is not satisfied. Then from Proposition 2 and (6) we have

$$
\frac{F_{1}(t)-F_{1}\left(a_{j}\right)}{F_{1}\left(a_{j+1}\right)-F_{1}\left(a_{j}\right)}<\frac{F_{2}(t)-F_{2}\left(a_{j}\right)}{F_{2}\left(a_{j+1}\right)-F_{2}\left(a_{j}\right)} .
$$


Define continuous and strictly increasing functions $h_{i}:[c, e) \rightarrow[0,1]$ by

$$
h_{i}(t)=\frac{F_{i}(t)-F_{i}(c)}{F_{i}(e)-F_{i}(c)}, i=1,2 .
$$

It follows from Proposition 2 that one of the inequalities

$$
h_{1}(t)>h_{2}(t), \quad h_{1}(t)<h_{2}(t)
$$

is satisfied for all $t \in(c, e)$. Suppose that $h_{1}(t)<h_{2}(t)$ for all $t \in(c, e)$. It follows from the continuity of the functions $h_{i}, i=1,2$ and the Darboux property that there exist numbers $t_{i} \in(c, e), i=1,2$, such that

$$
h_{1}(d)=1-h_{1}\left(t_{1}\right) \text { and } h_{2}\left(t_{2}\right)=1-h_{2}(d) .
$$

Thus, we have

$$
1-h_{2}\left(t_{2}\right)=h_{2}(d)>h_{1}(d)=1-h_{1}\left(t_{1}\right) \text { and then } h_{2}\left(t_{2}\right)<h_{1}\left(t_{1}\right) .
$$

The last inequality implies that $h_{2}\left(t_{2}\right)<h_{1}\left(t_{1}\right)<h_{2}\left(t_{1}\right)$ and hence the inequality $t_{2}<t_{1}$ must be satisfied. Multiplying the first equality (12) by $F_{1}(e)-F_{1}(c)$ and the second one by $F_{2}(e)-F_{2}(c)$ after simple calculations we obtain

$$
F_{1}(d)-F_{1}(c)=F_{1}(e)-F_{1}\left(t_{1}\right) \quad \text { and } \quad F_{2}\left(t_{2}\right)-F_{2}(c)=F_{2}(e)-F_{2}(d),
$$

which means that $\mu_{1}([c, d))=\mu_{1}\left(\left[t_{1}, e\right)\right)$ and $\mu_{2}\left(\left[c, t_{2}\right)\right)=\mu_{2}([d, e))$. It follows from Assumption 1 that $\mu_{i}\left(\left[t_{2}, t_{1}\right)\right)>0$ for all $i \in I$. Let $\left\{C_{i}\right\}_{i=1}^{n}$ be any partition of the interval $\left[t_{2}, t_{1}\right)$ into some subintervals satisfying $\mu_{i}\left(C_{i}\right)>0$ for all $i \in I$. Define new partition $\left\{A_{i}^{*}\right\}_{i=1}^{n} \in \mathscr{P}$ by

$$
A_{1}^{*}=\left(A_{1}^{0} \backslash[c, d)\right) \cup\left[t_{1}, e\right) \cup C_{1}, \quad A_{2}^{*}=\left(A_{2}^{0} \backslash[d, e)\right) \cup\left[c, t_{2}\right) \cup C_{2},
$$

and

$$
A_{k}^{*}=A_{k}^{0} \cup C_{k}, \quad \text { for } k=3, \ldots, n .
$$

Hence for all $i \in I$, we have

$$
\mu_{i}\left(A_{i}^{*}\right) \geq \mu_{i}\left(A_{i}^{0}\right)+\min _{i \in I}\left\{\mu_{i}\left(C_{i}\right)\right\}>\mu_{i}\left(A_{i}^{0}\right)
$$

which contradicts the fact that $\left\{A_{i}^{0}\right\}_{i=1}^{n}$ is an equitable optimal fair division. Then the inequality $h_{1}(t)>h_{2}(t)$ and also the inequality (11) must be satisfied which completes the proof of Lemma 1.

Lemma 2 Let $\left\{A_{i}^{0}\right\}_{i=1}^{n} \in \mathscr{P}$ be an equitable optimal fair division of the unit interval $[0,1)$. Assume that each $A_{i}^{\overline{0}}, i \in I$, is a finite union of intervals. Suppose that for fixed $j \in J$, and numbers $a_{j} \leq c_{1}<c_{2}<\cdots<c_{r} \leq a_{j+1}$, with $r \geq 4$ we have

$$
\left[c_{1}, c_{2}\right) \cup\left[c_{r-1}, c_{r}\right) \subset A_{i_{1}}^{0} \text { and }\left[c_{k}, c_{k+1}\right) \subset A_{i_{k}}^{0}, \text { for } k=2, \ldots, r-2
$$

for some $i_{k} \in I$. Then $i_{k}=i_{1}$ for all $k=2, \ldots, r-2$.

Proof Suppose that for some $k_{0} \in\{2, \ldots, r-2\}$ we have $i_{k_{0}} \neq i_{1}$. Without loss of generality we can assume that for neighbouring intervals $\left[c_{k}, c_{k+1}\right),\left[c_{k+1}, c_{k+2}\right)$,

$k=2, \ldots, r-3$ we have $i_{k} \neq i_{k+1}$. Otherwise we can connect intervals for which $i_{k}=i_{k+1}$ and we could consider fewer of such intervals. It follows from Lemma 1 that

$$
\sigma_{j}^{-1}\left(i_{1}\right)<\sigma_{j}^{-1}\left(i_{2}\right)<\cdots<\sigma_{j}^{-1}\left(i_{r-1}\right)<\sigma_{j}^{-1}\left(i_{1}\right) .
$$

This contradiction completes the proof. 
Proof of Theorem 3: First we observe that the NLP problem (7) has a solution. It is easy to check that the feasible set defined by (8) and (9) for $m(n-1)+1$ variables $\left\{z, x_{k}^{(j)} k=\right.$ $1, \ldots, n-1, j \in J\}$ is compact in $\mathbb{R}^{m(n-1)+1}$.

Suppose $\left\{A_{i}\right\}_{i=1}^{n}$ is an equitable optimal fair division. We show that there exist numbers $\left\{x_{k}^{(j)}\right\} k=1, \ldots, n-1, j \in J$, satisfying the following inequalities

$$
\begin{gathered}
0=a_{1} \leq x_{1}^{(1)} \leq \cdots \leq x_{n-1}^{(1)} \leq a_{2} \\
a_{2} \leq x_{1}^{(2)} \leq \cdots \leq x_{n-1}^{(2)} \leq a_{3} \\
\quad \cdots \\
a_{m} \leq x_{1}^{(m)} \leq \cdots \leq x_{n-1}^{(m)} \leq a_{m+1}=1
\end{gathered}
$$

and that

$$
A_{i}=\bigcup_{j=1}^{m}\left[x_{\sigma_{j}(i)-1}^{(j)}, x_{\sigma_{j}(i)}^{(j)}\right), i \in I .
$$

It follows from Assumption 2 that for any numbers $p_{i}, p_{k}$ and $i, k \in I, i \neq k$, the set $\left\{x \in[0,1): p_{i} f_{i}(x)=p_{k} f_{k}(x)\right\}$ is finite. Hence, by Theorem 2 each set $A_{i}$ must be a union of a finite number of intervals. Without loss of generality we may assume that all these intervals are left-closed and right-open. Hence each interval $\left[a_{j}, a_{j+1}\right), j \in J$, can be written as

$$
\left[a_{j}, a_{j+1}\right)=\bigcup_{l=1}^{q_{j}}\left[b_{l}^{(j)}, b_{l+1}^{(j)}\right), j \in J, l=1, \ldots, q_{j}
$$

with integers $q_{j} \geq 1$ and real numbers $b_{l}^{(j)}, b_{1}^{(j)}=a_{j}, b_{q_{j}+1}^{(j)}=a_{j+1}, j \in J, l=1, \ldots, q_{j}$, for which there exists $i \in I$ such that

$$
\left[b_{l}^{(j)}, b_{l+1}^{(j)}\right) \subset A_{i} .
$$

It follows from Lemma 2 that we can reduce (if necessary) the number of intervals $\left[b_{l}^{(j)}, b_{l+1}^{(j)}\right), j \in J, l=1, \ldots, q_{j}$ by finding numbers $x_{k}^{(j)}, j \in J, k=1, \ldots, n-1$ with $x_{k}^{(j)} \in\left\{b_{l}^{j}, l=1, \ldots, q_{j}\right\}$ such that

$$
A_{i} \cap\left[a_{j}, a_{j+1}\right)=\left[x_{\sigma_{j}(i)-1}^{(j)}, x_{\sigma_{j}(i)}^{(j)}\right) .
$$

If $A_{i} \cap\left[a_{j}, a_{j+1}\right)=\emptyset$ then we set $x_{\sigma_{j}(i)-1}^{(j)}=x_{\sigma_{j}(i)}^{(j)}$. Finally, we conclude that any equitable optimal partition $\left\{A_{i}\right\}_{i=1}^{n}$ takes the form (10) and the proof is complete.

The method presented in Theorem 3 can be used for obtaining also equitable $\varepsilon$-optimal fair divisions in case where the set $D$ defined by (2) is countably infinite. Then, for a given $\varepsilon>0$ there exists a partition $\left\{X_{1}, X_{2}\right\}$ of the unit interval $[0,1)$ such that

1. $X_{1}$ is a finite union of subintervals,

2. $D \cap X_{1}$ is finite,

3. for all $i \in I$ we have $\mu_{i}\left(X_{1}\right)>1-\varepsilon$.

There exist pairwise disjoint subintervals $X_{1}^{(j)}, j=1, \ldots, m$ of $[0,1)$, not necessary contiguous, such that $X_{1}=\cup_{j=1}^{m} X_{1}^{(j)}$ and for all $i, k \in I$ the ratios $\frac{f_{i}(x)}{f_{k}(x)}$ are strictly monotone 
on each $X_{1}^{(j)}$. Applying Theorem 3 for $X_{1}$ we obtain a partition $P^{\varepsilon}=\left\{A_{i}^{\varepsilon}\right\}_{i=1}^{n} \in \mathscr{P}_{e}\left(X_{1}\right)$ such that for all $i \in I$

$$
\mu_{i}\left(A_{i}^{\varepsilon}\right)=\delta\left(X_{1}\right) .
$$

Since $\delta=\delta\left(X_{1}\right)+\delta\left(X_{2}\right)$ and $\delta\left(X_{2}\right)<\varepsilon$ the partition $P^{\varepsilon}$ is equitable $\varepsilon$-optimal.

\section{Example}

Consider a problem of fair division for three players $I=\{1,2,3\}$ estimating measurable subsets of the unit interval $[0,1)$ using measures $\mu_{i}, i=1,2,3$, defined respectively by the following density functions

$$
f_{1}=12\left(x-\frac{1}{2}\right)^{2}, f_{2}=2 x, f_{3} \equiv 1, \quad x \in[0,1) .
$$

We use the algorithm described in Theorem 3 to obtain an equitable optimal fair division. First we need to divide the interval $[0,1)$ into some subintervals on which the densities $f_{i}, i=1,2,3$, separably satisfy SMLR property. For this reason we find the set $D$ defined by (2). It is easy to check that $D=\left\{\frac{1}{2}\right\}$ and hence by Proposition 1 the densities $f_{i}, i=$ $1,2,3$, satisfy the SMLR property on intervals $\left[0, \frac{1}{2}\right)$ and $\left[\frac{1}{2}, 1\right)$. Denote cumulative strictly increasing distribution functions by $F_{i}(t)=\int_{0}^{t} f_{i}(x) d x, i=1,2,3$. Then we have

$$
F_{1}(t)=4 t^{3}-6 t^{2}+3 t, F_{2}(t)=t^{2}, F_{3}(t)=t, \quad t \in[0,1) .
$$

Based on the inequalities (6) we establish the proper order of assigments of the subintervals $\left[0, \frac{1}{2}\right)$ and $\left[\frac{1}{2}, 1\right)$ to each player as follows: we take midpoints $\frac{1}{4}$ and $\frac{3}{4}$ of the two subintervals and verify that

$$
\frac{F_{1}(1 / 4)-F_{1}(0)}{F_{1}\left(\frac{1}{2}\right)-F_{1}(0)}>\frac{F_{3}(1 / 4)-F_{3}(0)}{F_{3}\left(\frac{1}{2}\right)-F_{3}(0)}>\frac{F_{2}(1 / 4)-F_{2}(0)}{F_{2}\left(\frac{1}{2}\right)-F_{2}(0)},
$$

and

$$
\frac{F_{3}(3 / 4)-F_{3}(0)}{F_{3}(1)-F_{3}\left(\frac{1}{2}\right)}>\frac{F_{2}(3 / 4)-F_{2}(0)}{F_{2}(1)-F_{2}\left(\frac{1}{2}\right)}>\frac{F_{1}(3 / 4)-F_{1}(0)}{F_{1}(1)-F_{1}\left(\frac{1}{2}\right)} .
$$

Hence, we obtain permutations

$$
\sigma_{1}=\left(\begin{array}{lll}
1 & 2 & 3 \\
1 & 3 & 2
\end{array}\right) \text { and } \sigma_{2}=\left(\begin{array}{lll}
1 & 2 & 3 \\
3 & 2 & 1
\end{array}\right)
$$

Now we are ready to formulate an NLP problem as in Theorem 3

$\max z$

subject to constraints

$$
\begin{aligned}
& z=4\left(\left(x_{1}^{(1)}\right)^{3}-\left(x_{2}^{(2)}\right)^{3}\right)-6\left(\left(x_{1}^{(1)}\right)^{2}-\left(x_{2}^{(2)}\right)^{2}\right)+3\left(x_{1}^{(1)}-x_{2}^{(2)}\right)+1 \\
& z=\left(x_{2}^{(2)}\right)^{2}-\left(x_{2}^{(1)}\right)^{2}-\left(x_{1}^{(2)}\right)^{2}+\frac{1}{4} \\
& z=x_{1}^{(2)}-x_{1}^{(1)}+x_{2}^{(1)}-\frac{1}{2}
\end{aligned}
$$


with respect to the variables $z,\left\{x_{k}^{(j)}\right\} k=1,2, j=1,2$, satisfying the following inequalities

$$
0 \leq x_{1}^{(1)} \leq x_{1}^{(2)} \leq \frac{1}{2} \leq x_{2}^{(1)} \leq x_{2}^{(2)} \leq 1 .
$$

Solving the above NLP problem using the Mathematica package we obtain

$$
\begin{aligned}
& z^{*} \approx 0.4843, x_{1}^{*(1)} \approx 0.1426, x_{1}^{*(2)}=a_{2}=0.5, x_{2}^{*(1)} \approx 0.6269, \\
& \quad x_{2}^{*(2)} \approx 0.9367 .
\end{aligned}
$$

Hence, we get the equitable optimal fair division $\left\{A_{i}^{*}\right\}_{i=1}^{3} \in \mathscr{P}$ of the unit interval $[0,1)$, where

$$
A_{1}^{*}=\left[0, x_{1}^{*(1)}\right) \cup\left[x_{2}^{*(2)}, 1\right), \quad A_{2}^{*}=\left[x_{2}^{*(2)}, x_{2}^{*(1)}\right) \text { and } A_{3}^{*}=\left[x_{2}^{*(1)}, x_{1}^{*(1)}\right) .
$$

Acknowledgements The author would like to thank the referee for very careful review and constructive comments which substantially helped improving the quality of the paper.

Open Access This article is distributed under the terms of the Creative Commons Attribution 4.0 International License (http://creativecommons.org/licenses/by/4.0/), which permits unrestricted use, distribution, and reproduction in any medium, provided you give appropriate credit to the original author(s) and the source, provide a link to the Creative Commons license, and indicate if changes were made.

\section{References}

Aubin, J. P. (1980). Mathematical methods of game and conomic theory. Amsterdam: North-Holland Publishing Company.

Beckenbach, E. F. (1937). Generalized convex functions. Bulletin of the American Mathematical Society, 43, 363-371.

Dall'Aglio, M., \& Luca, Di. (2014). Finding maxmin allocations in cooperative and competitive fair division. The Annals of Operations Research, 223, 121-136.

Dall'Aglio, M., \& Luca, Di. (2015). Bounds for $\alpha$-opimal partitioning of a measurable space based on several efficient partitions. The Journal of Mathematical Analysis and Applications, 425, 854-863.

Dall'Aglio, M., Legut, J., \& Wilczyński, M. (2015). On finding optimal partitions of a measurable space. Mathematica Applicanda, 43(2), 157-172.

Dvoretzky, A., Wald, A., \& Wolfowitz, J. (1951). Relations among certain ranges of vector measures. Pacific Journal of Mathematics, 1, 59-74.

Elton, J., Hill, T., \& Kertz, R. (1986). Optimal partitioning ineaqualities for non-atomic probability measures. Transactions of the American Mathematical Society, 296, 703-725.

Hill, T., \& Tong, Y. (1989). Optimal-partitioning ineaqualities in classification and multi hypotheses testing. The Annals of Statistics, 17, 1325-1334.

Jessen, B. (1931). Bemaerkiner om konvekse functioner og uligheder imellem midelvaerdier, Matematisk tidsskfift. B., 17-28 .

Karlin, S., \& Novikoff, A. (1963). Generalized convex inequalities. Pacific Journal of Mathematics, 13, 12511279.

Knaster, B. (1946). Sur le probleme du partage pragmatique. de H. Steinhaus. Annales de le Societé Polanaise Mathematique, 19, 228-230.

Legut, J. (1988). Inequalities for $\alpha$-optimal partitioning of a measurable space. Proceedings of the American Mathematical Society, 104, 1249-1251.

Legut, J. (2017). Optimal fair division for measures with piecewise linear density functions. International Game Theory Review, 19(2), 1750009.

Legut, J., \& Wilczyński, M. (1988). Optimal partitioning of a measurable space. Proceedings of the American Mathematical Society, 104, 262-264.

Legut, J., \& Wilczyński, M. (2012). How to obtain a range of nonatomic vector measure in $\mathbb{R}^{2}$. The Journal of Mathematical Analysis and Applications, 394, 102-111. 
Palmer, J. A. (2003). Relative convexity. Unpublished paper.

Popoviciu, T. (1936). Notes sur les fonctions d'ordre superieur. Mathematica, 12, 81-92.

Shisha, O., \& Cargo, G. T. (1964). On comparable means. Pacific Journal of Mathematics, 3(14), $1053-1058$. 